\section{Earthquake damage}

SIR-Tiedemann ${ }^{1}$ has recently commented on our paper ${ }^{2}$ on the resonance effects of the September 1985 earthquakes in Mexico City. Some of his comments and proposed 'rules' are quite relevant and deserve a detailed answer. However, in his opening statement he implies that the distribution of damage simply follows the geographical distribution of 5-15 storey buildings, thus implying that our conclusions are invalid. This is not true. Conservatively speaking, over two-thirds of the 5-15 storey structures were virtually unscathed and even within the lake region of Mexico City there are whole areas with a high density of such structures that were only lightly hit. For example, the Cuauhtemoc area has a larger concentration of ten storey buildings than Roma and there was not nearly as much damage. Furthermore, areas with no high buildings but with "small, old and vulnerable structures" did sometimes suffer great destruction. One case is the Tepito area where such buildings collapsed in great number. (Tepito corresponds to letter B in ref. 2 , Fig. 1). We predicted this to be the centre of highest acceleration (see Fig. 2 in ref. 2).

Tiedemann does raise some pertinent points about our paper. The eastern limits of the extinct lake are not well defined, as we emphasized', but recently the proposal of a sunken mountain range (Sierrita del Peñón), that essentially coincides with our proposed limits, has been forwarded ${ }^{3}$. We are aware that the composition of the different deposit layers is not homogeneous and this has to be taken into account; we are currently working on this. The contrast between the soft-ground area and the surrounding volcanoes and hills is, however, far greater than the changes within the former which should only provide refining factors.

It is indeed deplorable that so few accelerographs were available. The only apparatus located within the ancient lake area (letter $G$ of ref. 2, Fig. 1) shows a striking dominance of the 2-s period (ref. 2, Fig. 2).

We also agree with Tiedemann that earthquake damage is controlled by many parameters. As a consequence, all new ideas should be carefully examined and not hurriedly discarded. We insist that we do not imply that we can explain the damage pattern only by the interference pattern. This impression may have stemmed from some comments on our work that overemphasize our statements (see for instance ref. 4). In ref. 2 we report that both pressure and shear waves are transmitted at the old lake's interphase and while the former produce interference patterns the latter cover the whole area uniformly, adding significantly to the tremor effects. In any case our present model gives a useful first approximation to the interference pattern. Furthermore, we are now investigating a more realistic model $^{2}$ that will predict the directions of ground motion within the relevant area, a prediction that may be tested in future earthquakes for which a much more reasonable amount of seismographic data should be available. At a time when human lives are dependent on construction and protection of buildings, the neglect of any fact (theoretical or empirical) is, as Tiedemann states, dangerous.

J. FLores

O. NOVARO

Instituto de Física,

UNAM, AP20-364,

Mexico 01000 DF, Mexico

1. Tiedemann. H. Nature 329, 677 (1987)

2. Flores, J., Novaro, O. \& Seligman, T.H. Nature 326, $783-$ $785(1987)$.

Alvarez, R. Engineering 1, (abstr. 1.5) 107-108 (1986) New Scientist 28, 7 May (1987)

\section{Glycine vs GABA receptors}

SiR-Comparison of the primary structures of the strychnine-binding subunit of the glycine receptor ${ }^{1}$ and both subunits of the $\mathrm{GABA}_{\mathrm{A}}$ receptor ${ }^{2}$ has revealed the existence of a gene family for neurotransmitter-gated ion channel receptors which comprises both anionic and cationic chan-

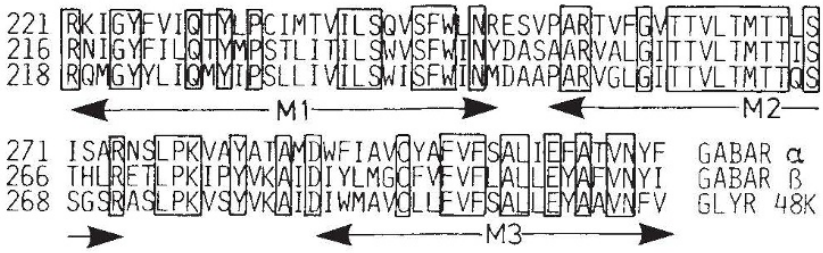

residues (TTVLTMTT) are invariant within M2. As segment M2 is thought to line the channel lumen of the different ion-conducting neurotransmitter receptors ${ }^{1,2,4-6}$ we suggest that this conserved sequence will be characteristic of all ligand-gated chloride channels.

Third, a large number of positivelycharged amino-acid residues occurs in the putative chloride channel-mouth domains of both glycine and $\mathrm{GABA}_{\mathrm{A}}$ receptor polypeptides. These clustered charges have been implicated in anion binding and channel selectivity ${ }^{1,2}$ and may constitute a general feature of ligand-gated anion channels.

Finally, chloride-conducting neurotransmitter receptor proteins have no amphipathic $\alpha$-helix preceding the fourth hydrophobic region, M4. Such an amphipathic helix is found in all $n A C h R$ subunits, and was originally proposed to form the inner wall of the cation channel? The above and further comparisons within this receptor family have been made in more detail in an article appearing elsewhere ${ }^{8}$.

These conserved features indicate a late separation of $\mathrm{GABA}_{\mathrm{A}}$ and glycine receptors during ligand-gated ion-channel evolution. In the case of these inhibitory channels, the acquisition of ion selectivity probably preceded that of agonist specificity. However, domain exchanges by exon shuffling may also have contributed to the generation of the present ion channel receptor family, since cationconducting GABA receptors and anionselective nAChRs have been described in dif-

Alignment of transmembrane domains MI to M3 of the GABA and ferent systems 9 . Seqglycine receptor polypeptides. Identical amino-acid residues are boxed. uence and structural nels including the nicotinic acetylcholine receptor (nAChR) and presumably those gated by amino acids involved in excitatory neurotransmission, such as glutamate. Sequence comparison shows that the subunits of all known members of this gene family have the same number and distribution of membrane-spanning helices, resulting in an identical transmembrane topology $y^{1-3}$. The first transmembrane region predicted, M1, contains a proline residue at an identical position pointing to a common mechanism of channel gating ${ }^{1,2}$

In addition, the subunits of the glycine and $\mathrm{GABA}$ receptor display some striking structural similarities. First, their sequences are considerably more similar to each other $(34 \%-38 \%)$ than they are to the subunits of nAChRs $(15 \%-20 \%)$. Second, the $\mathrm{GABA}_{\mathrm{A}}$ and glycine receptors are most similar within the first three membrane-spanning regions (see figure); eight contiguous amino-acid information on these latter receptors should contribute to our understanding of how diversity was generated within this important super-family.

Gabriele Grenningloh ECKART GUNDELFINGER BERTRAM SCHMITT HeINRICH BetZ Molecular Neuropharmacology Lab, ZMBH, University of Heidelberg, 6900 Heidelberg, FRG

Mark G. Darlison ERIC A. BARNARD MRC Molecular Neurobiology Unit, MRC Centre,

Cambridge CB2 2QH, UK

Peter R. SChofield Peter H. Seeburg

Laboratory of Molecular

Neuroendocrinology

$Z M B H$, University of Heidelberg, 6900 Heidelberg, FRG

Grenningloh, G. et al. Nature 328, $215-220$ (1987). Schofield. P. et al. Nature 328, 221-226 (1987) 